\title{
Effect of preservative-free and preserved prostaglandin analogues on the histology of cornea of adult male guinea pigs following repeated exposure
}

\author{
A.F. Ali' ${ }^{1}$, R.M. Salama²(1), M.A. Soliman ${ }^{1}$ \\ ${ }^{1}$ Department of Histology, Faculty of Medicine, Menoufia University, Egypt \\ 2Department of Anatomy and Embryology, Faculty of Medicine, Menoufia University, Egypt
}

[Received: 14 August 2020; Accepted: 25 November 2020; Early publication date: 23 December 2020]

Background: Glaucoma is a group of eye diseases that can cause vision loss. Prostaglandin analogues (PGAs) are known to be first-line treatment for patients with glaucoma. Latanoprost is a good, efficient and well-tolerated PGA that is currently available as latanoprost with benzalkonium chloride (BAC) (Xalatan) and preservative-free (PF) prostaglandin analogue latanoprost (Monopost). Lately, using PF anti-glaucoma agents has been considered an essential procedure for enhancing glaucoma care. This study aimed to analyse the histological changes within the corneal tissue with the use of currently available preserved prostaglandins-derived eye drops and PF prostaglandin analogue.

Materials and methods: In this study, 40 male guinea pigs were divided into four equal groups. Control group, Latanoprost with 0.02\% BAC-treated group, Recovery group and PF latanoprost-treated group. After 2 months, the corneal tissues of guinea pigs were prepared for light and electron microscopic studies; morphometric and statistical studies were performed.

Results: Our results indicated that guinea pigs treated with latanoprost with $B A C$ exhibited ocular surface changes: there was epithelial thinning with desquamation, the stroma showed irregularly arranged collagen fibres and small keratocytes. Morphometrically, there was a marked decrease in the thickness of epithelium and number of keratocytes. Negative periodic acid Schiff (PAS) reaction was observed in some parts of the epithelial basement membrane. The epithelium gave a strong positive immunoreactivity for BCl-2-associated X protein (BAX). Guinea pigs left to recover exhibited improvement, while treatment of animals with PF latanoprost resulted in nearly normal corneal structure.

Conclusions: In conclusion, PF prostaglandin anti-glaucoma medication seems to be better and have protective effect on cornea of male guinea pigs than prostaglandins with BAC preservative. (Folia Morphol 2022; 81, 1: 52-64)

Key words: glaucoma, cornea, guinea pigs, prostaglandins analogues, periodic acid Schiff (PAS), Bcl-2-associated X protein (BAX) 


\section{INTRODUCTION}

Glaucoma is an optic neuropathy that is considered the main cause of blindness in the world, affecting approximately 67 million people worldwide $[6,30]$. Glaucoma often occurs due to elevated intraocular pressure (IOP) that leads to damage of the optic nerve which can progress to irreversible vision loss, when left without treatment [36].

Intraocular pressure is an essential risk factor and is the main aim for the currently approved glaucoma treatment through the use of topical ocular hypotensive drops [21] that should be taken through life to inhibit or stop loss of retinal ganglion cells and visual impairment [31].

Prostaglandin analogues (PGAs) are one of the most important drugs utilised for ocular hypertension and glaucoma treatment [26]. These compounds are known to be highly effective in decreasing IOP [19].

Uzunel [39] reported that PGAs stimulated drainage of aqueous humour through the uveo-scleral outflow pathway and facilitated the trabecular outflow resulting in reducing IOP. Five different prostaglandins (PGs) are known to be used for treatment of glaucoma, namely, isopropyl unoprostone, latanoprost, travoprost, bimatoprost, and tafluprost [16].

Long-term local treatment with these anti-glaucoma drugs is always associated with ocular surface disease such eye colour change, darkening of eyelid skin, droopy eyelids, sunken eyes, eye redness, and itching [32].

Kim et al. [16] referred these side effects to the components of these topical hypotensive drops such as benzalkonium chloride (BAC) preservative. BAC is the most commonly applied preservative in eye drops having highly effective anti-microbial features. It is a quaternary ammonium salt, a cationic surfactant and tension-active compound. It breaks up the lipid layer of tear film and cell membranes of corneal surface. BAC acts also by denaturing proteins and disrupting the cell membrane, thus improving the active compounds penetration; so, it could be employed as a penetration enhancer $[2,25]$. It has the advantage of being relatively well tolerated with few allergic side effects [6]. Having very slow turnover, BAC remains within the ocular tissues after a single drop administration about 48 hours [16].

BAC side effects seemed to be time- and dose-dependent, rising with larger amounts utilised over prolonged periods $[22,42]$. With rising attention to the ocular surface side effects of this preservative, other types of preservatives-containing PGs and new preservative-free (PF) PGs have been advanced [38].

So, this current study was designed to analyse the histological alterations within the corneal tissue with the use of currently available (PGs)-derived eye drops, namely latanoprost with BAC (Xalatan) and PF PGA latanoprost (Monopost), in male guinea pigs.

\section{MATERIALS AND METHODS}

Animals and experimental protocol

The present experimental study was conducted at Research Centre in Menoufia University, Egypt. Study protocol was reviewed and observed via the Animal Ethics Committee of the Research Laboratory of Experimental Animals at Faculty of Medicine, Menoufia University, Egypt. Forty male adult guinea pigs, weighting 400-450 $\mathrm{g}$ were employed in the current work. They were kept in cages at room temperature. The water and food were available. Strict hygiene was followed to keep a healthy medium for the guinea pigs.

\section{Animal groups}

The animals were distributed randomly into four groups, each included 10 guinea pigs:

- group I (Control group): the guinea pigs were left without treatment during the experimental period;

- group II (Latanoprost with BAC treated group): they were treated topically with latanoprost preserved with $0.02 \%$ benzalkonium chloride $(0.005 \%$ Xalatan $125 \mu \mathrm{g} / 2.5 \mathrm{~mL}$, Pfizer, New York, USA);

- group III (Recovery group): they were treated as group II and left for 2 weeks without treatment for recovery;

- group IV (PF latanoprost-treated group): the guinea pigs in this group were treated topically with preservative free latanoprost (Monopost $50 \mu \mathrm{g} / \mathrm{mL}$, Thea Pharmaceuticals Ltd., UK).

All guinea pigs received treatment as a single eye drop (1.5 $\mu \mathrm{g}$ latanoprost) in both eyes daily for 2 months [14].

At the assigned time of scarification for each group, cardiac perfusion was done under anaesthesia using sodium pentobarbital, $40 \mathrm{mg} / \mathrm{kg}$ by intraperitoneal injection [15]. From each animal, both eyeballs were enucleated rapidly and the corneal tissues were prepared for light and transmission electron microscopic studies. 


\section{Light microscopic study}

From each animal, the right eye ball was fixed in $10 \%$ formaldehyde solution and processed for paraffin sections of about $7 \mu \mathrm{m}$ thick. Sections were obtained and stained with haematoxylin and eo$\sin (\mathrm{H} \& E)$ to demonstrate the histological details and Mallory's trichrome stain to detect the collagen fibres [3]. For histochemical study, the corneal sections were processed to be stained by periodic acid Schiff (PAS) reaction for determination of carbohydrate (epithelial basement membrane) [3]. For immunohistochemical study, Bcl-2-associated $X$ protein (BAX) immune-staining was carried out (a marker of apoptosis), paraffin sections were put within monoclonal BAX antibody (Ab-14 Golden, Lab Vision Clone B-9; Santa Cruz Biotechnology Inc., USA). Negative control sections were processed by replacing the primary antibody with buffer alone. Hodgkin's lymphoma was used as a positive control. Brown cytoplasmic staining was scored as a positive reaction. Counterstaining was performed by using Meyer's haematoxylin [29].

\section{Transmission electron microscopic study}

The cornea of the left eye was dissected. Small corneal specimens were obtained and rapidly fixed in $3 \%$ glutaraldehyde with $0.1 \mathrm{M}$ phosphate buffer at $\mathrm{pH} 7.4$, and then, processed for examination, using a Jeol electron microscope (Seo-Russia) in Tanta E.M. Centre at Faculty of Medicine, Tanta University [12].

\section{Morphometric study and statistical analysis}

From each animal in all groups, the cornea was examined under light microscope using objective lens $\times 40$. Thickness of corneal epithelium in $\mu \mathrm{m}$ and keratocytes number was measured in 10 high power fields in each specimen.

The morphometric results were analysed and compared by Student's t-test. P-value was utilised to estimate the significant change in each parameter in the experimental animals in comparison with the control group. Then, the data were tabulated as mean \pm standard deviation and then, analysed utilising statistical package for the Social Science Software (SPSS). P value was set at $0.05 ; p>0.05$ was considered non-significant, $p$ value $<0.05$ was considered significant and $p$ value $<0.001$ was considered highly significant [28].

\section{RESULTS}

Effects of preservative-free and preserved prostaglandin analogues on corneal morphology

Seven $\mu \mathrm{m}$ paraffin corneal sections stained with $\mathrm{H} \& \mathrm{E}$ of the different experimental groups to analyse the histological alterations with the use of latanoprost with BAC (Xalatan) and PF PGA latanoprost (Monopost) in male guinea pigs was done. Group I (Control group) showed well defined corneal layers, these were: the outer epithelium, Bowman's membrane, stroma, Descemet's membrane and inner endothelium. The corneal epithelium was stratified squamous non-keratinised epithelium with smooth upper surface. It consisted of basal layer of single columnar cells with basal oval nuclei, intermediate layer consisting of polyhedral cells with central rounded nuclei arranged in 2 to 3 rows and superficial layer consisting of one to two rows of flattened squamous cells with flat nuclei. Under the epithelium, an acidophilic homogenous membrane appeared, Bowman's membrane. The stroma constituting the most corneal thickness was formed of bundles of collagen fibres which were arranged regularly and keratocytes appeared as flat cells with flattened nuclei, scattered between collagen fibres (Fig. 1A). While, the corneal sections from group II (Latanoprost with BAC treated group) exhibited epithelial thinning with flat nuclei (Fig. 1B). The thickness of corneal epithelium showed a highly significant decrease as compared with control group ( $p<0.001$ ) (Table 1, Fig. 3A). Some surface epithelial cells were exfoliated and desquamated and other epithelial cells exhibited vacuolated cytoplasm and their nuclei revealed variable degrees of degeneration, karyolysis and pyknosis (Fig. 1C). The stroma demonstrated irregularly arranged collagen fibres which were separated by wide spaces (Figs. 1B, C) and small dark keratocytes were seen (Fig. 1C). The mean number of keratocytes was highly significantly decreased in comparison with the control group ( $p<0.001$ ) (Table 1, Fig. 3B). Cellular infiltration, neovascularisation (Fig. 2B) and congested blood vessels (Fig. 1C) were also observed in the stroma. Group III (Recovery group) revealed less degenerative changes in the form of slight superficial epithelial cells desquamation and corrugated basement membrane. The stroma consisted of collagen bundles arranged regularly and spindle-shaped keratocytes with pale nuclei in between. Some keratocytes appeared small in size 

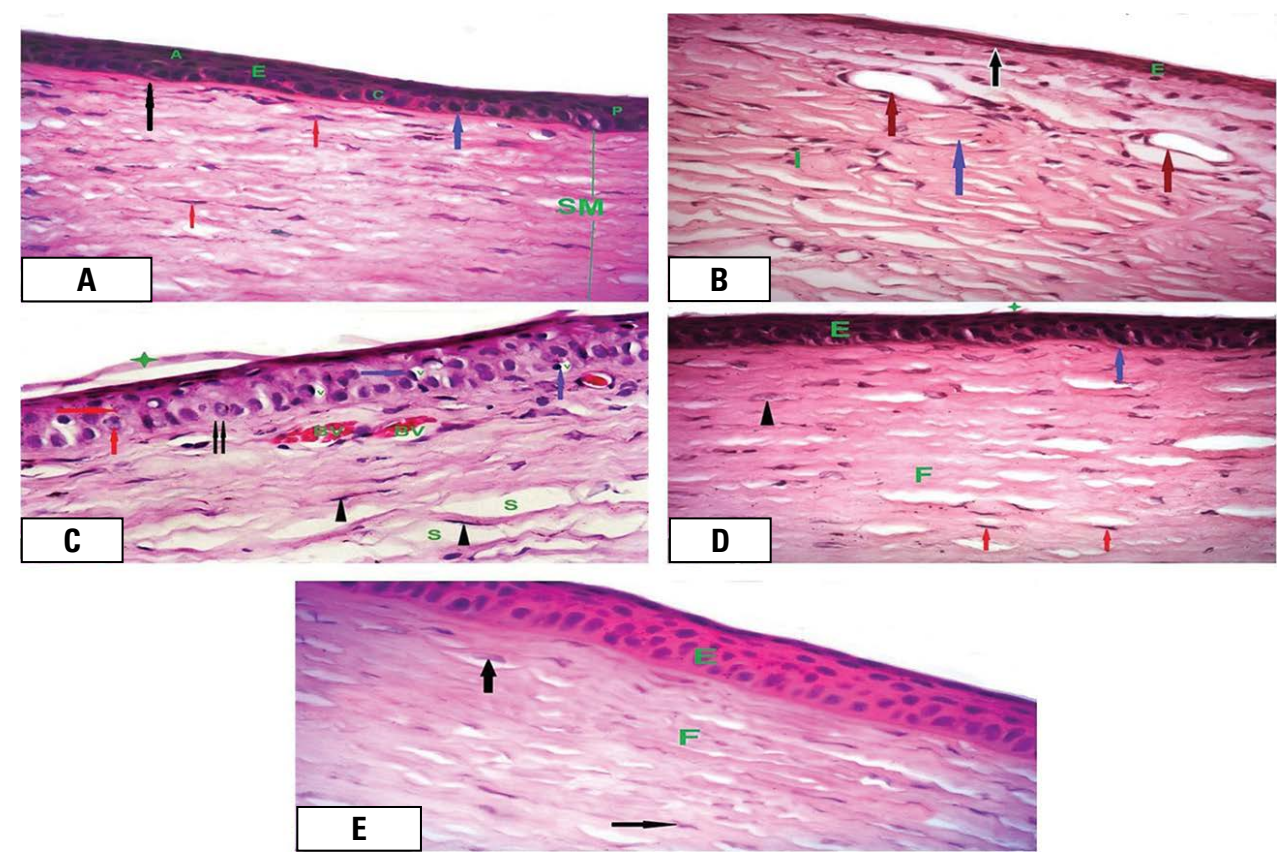

Figure 1. Photomicrographs of haematoxylin and eosin (H\&E) stained corneal sections of the different experimental groups; A. Control group: illustrating non-keratinized stratified squamous epithelium $(\mathrm{E})$ resting on clear basement membrane (blue arrow) and consists of basal layer of columnar cells (C) with basal oval nuclei, intermediate layers of polyhedral cells $(\mathrm{P})$ with central rounded nuclei and superficial layer of flattened cells (A) with flat nuclei. The Bowman's membrane (black arrow) appears as an acidophilic homogenous layer under the epithelium. The stroma (SM) consists of collagen fibres arranged regularly with spindle-shaped keratocytes with flat nuclei (red arrows) in-between; B. Group II: illustrating all epithelial layers (E) appear with flattened nuclei (black arrow). The stroma shows irregularly arranged collagen fibres (blue arrow) and neovascularisation (red arrows). Notice, cellular infiltration (I) within the stroma; C. Group II: illustrating desquamation of the superficial epithelium (star) is observed, the epithelial cells appear with vacuolated cytoplasm (V) and their nuclei show variable degrees of degeneration, karyolysis (red arrows) and pyknosis (blue arrows). The stroma shows wide spaces (S) in between collagen fibres, congested blood vessels (BV) and small dark keratocytes (arrow heads). Notice, discontinuation of basement membrane (double arrow); D. Group III: illustrating stratified squamous non-keratinised epithelium (E) resting on corrugated basement membrane (blue arrow). The superficial cells appear with slight desquamation (star). The stroma is formed of parallel arranged collagen fibres $(F)$ with spindle shaped keratocytes with pale nuclei in between (arrow head). Notice, some keratocytes appear small with condensed nuclei (red nuclei); E. Group IV: illustrating preserved corneal epithelium (E). The stroma appears with regularly arranged collagen fibres $(\mathrm{F})$ with keratocytes (arrows) in between. $\mathrm{H} \& \mathrm{E} \times 400$.

Table 1. Comparison between control group and other studied groups

\begin{tabular}{|c|c|c|c|c|c|}
\hline & Group I & Group II & Group III & Group IV & $P$ value \\
\hline Corneal epithelial thickness & $41.1 \pm 1.2$ & $38.4 \pm 3.8$ & $26.1 \pm 1.2$ & $41.2 \pm 1.2$ & $\begin{array}{l}\mathrm{P} 1=0.000 \\
\mathrm{P} 2=0.010 \\
\mathrm{P} 3=0.713 \\
\mathrm{P} 4=0.000\end{array}$ \\
\hline Number of keratocytes & $23.7 \pm 0.6$ & $20.9 \pm 5.2$ & $16.5 \pm 2.5$ & $24.3 \pm 1.7$ & $\begin{array}{l}\mathrm{P} 1=0.000 \\
\mathrm{P} 2=0.022 \\
\mathrm{P} 3=0.157 \\
\mathrm{P} 4=0.000\end{array}$ \\
\hline
\end{tabular}

P1 — comparison was done between group 1 (Control group) and group II (Latanoprost with BAC-treated group); P2 — comparison was done between group I (Control group) and group III (Recovery group); P3 - comparison was done between group I (Control group) and group IV (PF latanoprost-treated group); P4 - comparison was done between group IV (PF latanoprost group) and group II (Latanoprost with BAC-treated group); $p<0.001$ was considered highly significant; $p<0.05$ was considered significant; $p>0.05$ was considered non-significant

with condensed nuclei (Fig. 1D). The corneal epithelial thickness and the mean number of keratocytes were improved compared with group II $(p<0.05)$ (Table 1, Fig. 3A, B). Group IV (PF latanoprost treated group) revealed better corneal structure that appeared almost similar to that of the control group (Fig. 1E). There was a nonsignificant difference $(p>0.05)$ in corneal epithelial thickness and the 

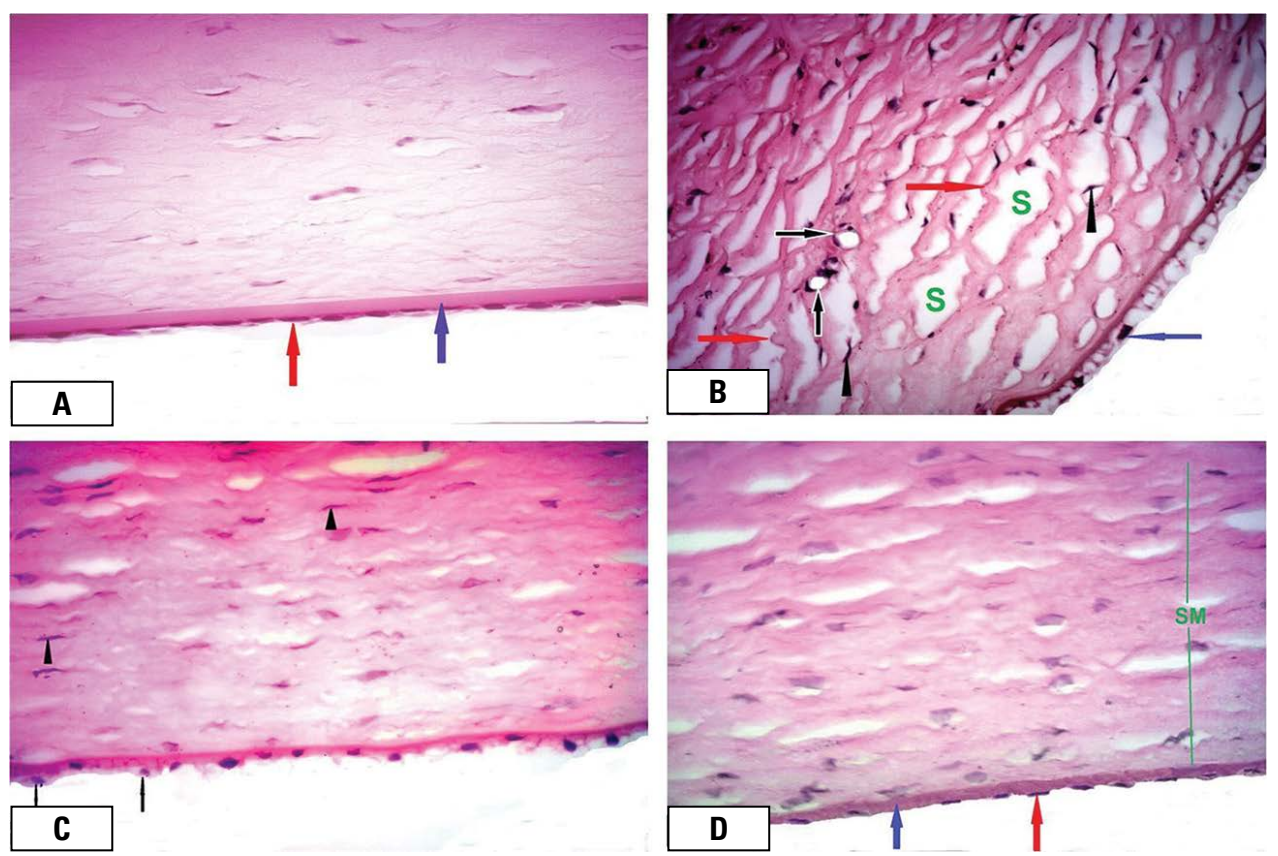

Figure 2. Photomicrographs of haematoxylin and eosin (H\&E) stained corneal sections of the different experimental groups; A. Control group: illustrating the posterior aspect of the cornea Descemet's membrane (blue arrow) appears as a homogenous acellular membrane and lined by a single layer of endothelial cells with flat nuclei (red arrow); B. Group II: illustrating apparently few endothelial cells with small pyknotic nuclei (blue arrow). The stroma appears with irregularly arranged collagen fibres (red arrows) with wide spaces in between (S). Small keratocytes (arrow heads) with dark nuclei are observed. Note, appearance of neovascularisation (black arrows) in the stroma; C. Group III: illustrating some endothelial cells (arrows) separated from the Descemet's membrane. Notice, some small-sized keratocytes (arrow heads) with condensed nuclei in the stroma; D. Group IV: illustrating Descemet's membrane (blue arrow) which appears as a homogenous acellular membrane and lined by a single layer of endothelial cells (red arrow). Notice, normal appearance of stroma (SM). H\&E $\times 400$.
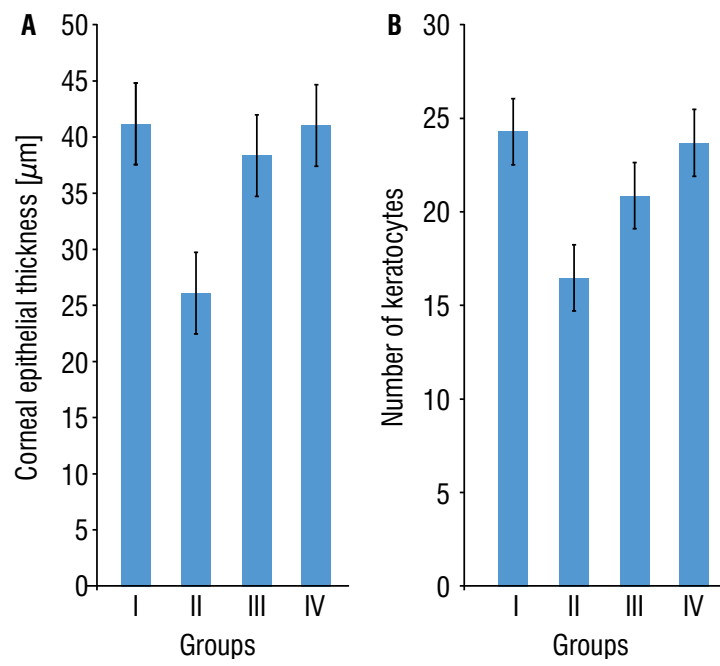

Figure 3. A histogram showing mean values of the corneal epithelial thickness (A) and keratocyte number $(\mathbf{B})$ of the different experimental groups.

mean number of keratocytes when compared with control group. While at the same time, these animals showed a highly significant decrease in these parameters in comparison with group II $(p<0.001)$ (Table 1, Fig. 3A, B). Regarding to the posterior as- pect of the cornea, group I displayed flat endothelial cells arranged in one layer and supported via a homogenous acellular Descemet's membrane (Fig. 2A). Group II showed apparently few endothelial cells with small pyknotic nuclei. Stroma with irregularly arranged collagen fibres and with wide spaces in between was seen. The keratocytes appeared small in size with dark nuclei (Fig. 2B). Group III revealed few endothelial cells separated from the Descemet's membrane (Fig. 2C). Moreover, group IV displayed acellular Descemet's membrane lined by a single layer of endothelial cells (Fig. 2D).

In order to visualise changes in collagen fibre architecture Mallory trichrome-stained corneal sections of the different experimental groups were performed. Group I (Control group) revealed regularly arranged collagen fibres (Fig. 4A). However, in group II (Latanoprost with BAC treated group), there were widely separated and irregularly arranged collagen fibres (Fig. 4B). Group III (Recovery group) exhibited regularly arranged collagen fibres. Wide space between collagen fibres was observed (Fig. 4C). In group IV (PF latanoprost treated group), there was regular arrangement of collagen fibres similar to control (Fig. 4D). 

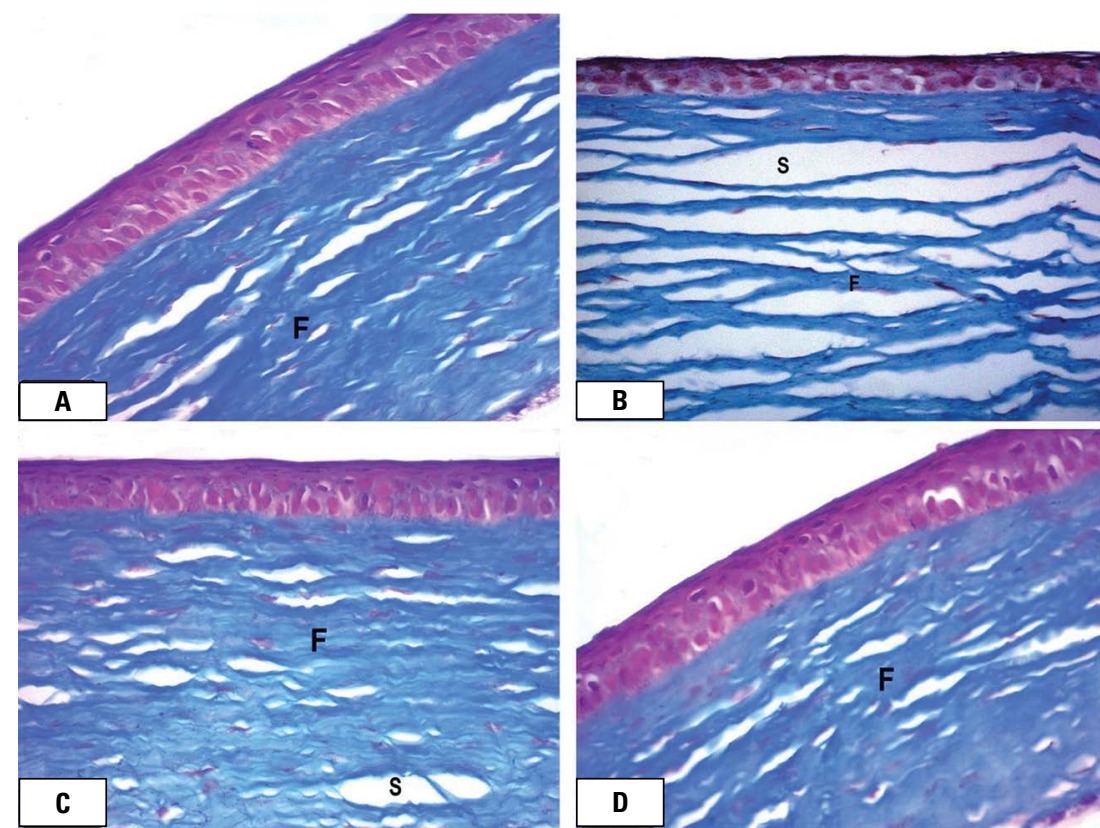

Figure 4. Photomicrographs of Mallory's trichrome-stained corneal sections of the different experimental groups; A. Control group: illustrating regularly arranged collagen fibres (F); B. Group II: illustrating, widely separated (S) and irregularly arranged collagen fibres (F); C. Group III: illustrating, regularly arranged collagen fibres (F). Notice, wide space between collagen fibres (S); D. Group IV: illustrating, regular arrangement of collagen fibres (F) similar to control group. Mallory's trichrome $\times 400$.
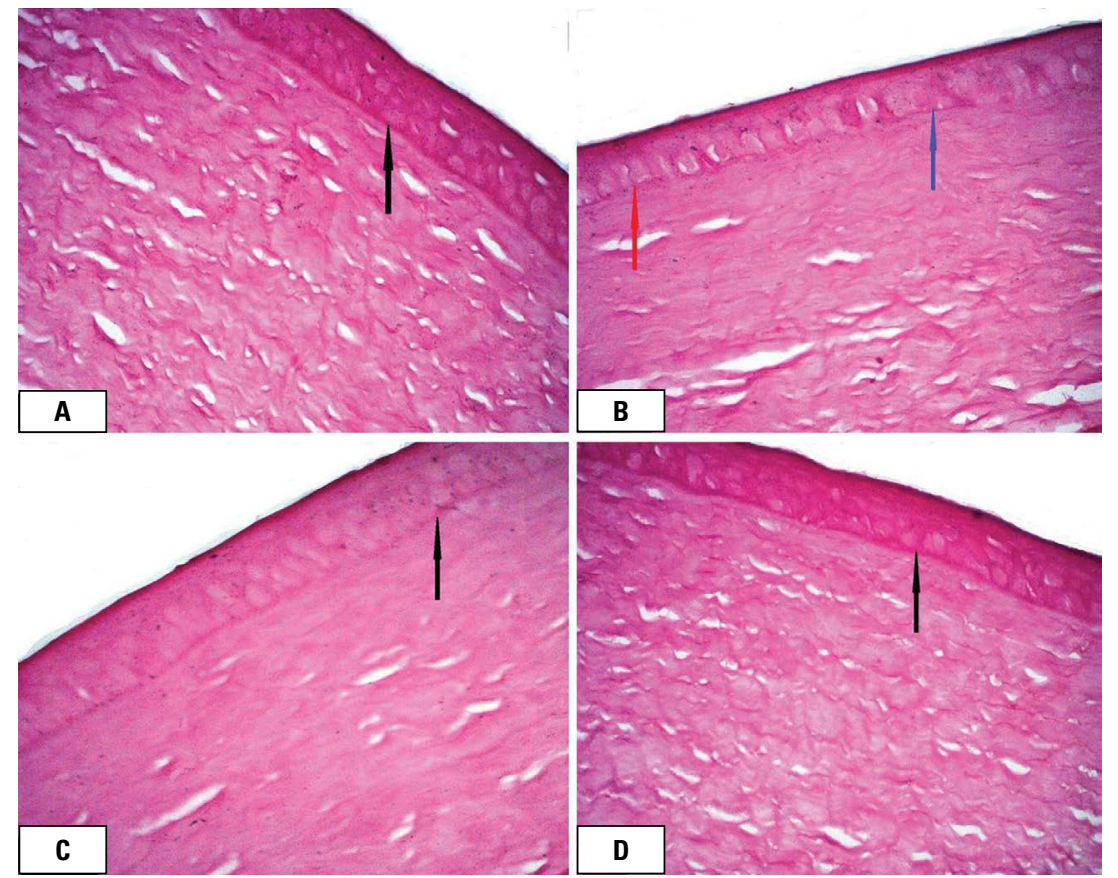

Figure 5. Photomicrographs of periodic acid Schiff (PAS) stained corneal sections of the different experimental groups; A. Control group: illustrating strong positive PAS reaction in the epithelial basement membrane (arrow); B. Group II: illustrating, mild positive PAS reaction in some parts (blue arrow) and negative in other parts (red arrow) of the epithelial basement membrane; C. Group III: illustrating, mild positive PAS reaction in the basement membrane (arrow); D. Group IV: illustrating, strong positive PAS reaction in the basement membrane (arrow). PAS $\times 400$.
For determination of carbohydrate (epithelial basement membrane), PAS stained corneal sections of the different experimental groups were done. Group I (Control group) exhibited strong positive reaction in the epithelial basement membrane (Fig. 5A). However, mild positive PAS reaction in some parts and negative in other parts of the basement membrane were seen in group II (Latanoprost with BAC treated group) (Fig. 5B). Group III (Recovery group) revealed mild positive reaction (Fig. $5 \mathrm{C}$ ). In group IV (PF latanoprost treated group), there was strong positive PAS reaction in the basement membrane similar to control group (Fig. 5D).

For detection of apoptosis immunohistochemically in corneal tissue, BAX immune-marker expression was determined. There was a negative BAX expression in the corneal tissue of group I (Fig. 6A), whereas in group II, strong positive cytoplasmic BAX reaction 

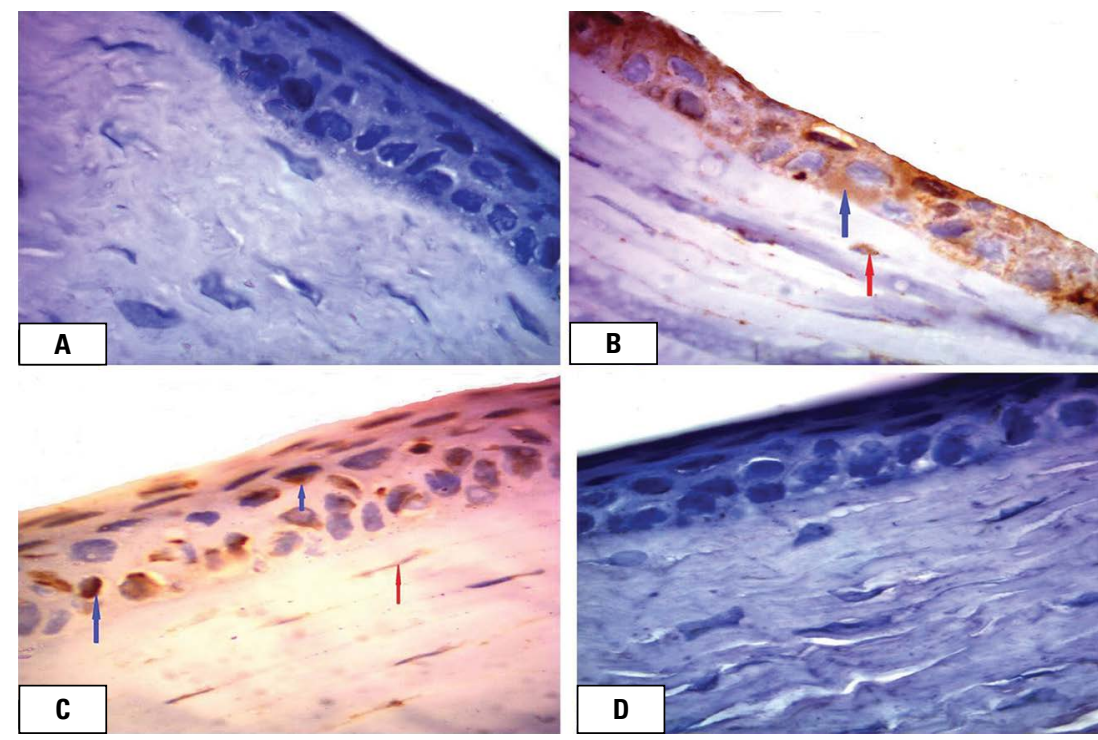

Figure 6. Photomicrographs of Bcl-2-associated $X$ protein $(B A X)$ immune-stained corneal sections of the different experimental groups; A. Control group: illustrating negative $B A X$ reaction in the epithelium and stroma; B. Group II: illustrating, strong positive cytoplasmic BAX reaction in the epithelium (blue arrow) and mild reaction (red arrow) in the keratocytes; C. Group III: illustrating, moderate BAX reactivity in the corneal epithelial cells (blue arrows) and mild reaction in the keratocytes (red arrow); D. Group IV: illustrating, negative $B A X$ reaction in the epithelium and stroma. BAX $\times 1000$. in the epithelium and mild reaction in keratocytes were observed (Fig. 6B). However, group III, exhibited moderate $B A X$ reactivity in the corneal epithelial cells and mild reaction in keratocytes (Fig. 6C). In group IV, there was negative reaction in the epithelium and stroma similar to control animals (Fig. 6D).

\section{Effects of preservative-free and preserved prostaglandin analogues on corneal ultrastructure}

Electron microscopic examination was done to confirm the light microscopic findings.

Group I (Control group), the corneal epithelium had basal columnar cells with euchromatic rounded nuclei. Hemi-desmosomes appeared as electron dense spots and were seen on the basal sides of these cells, facing the basement membrane. The intermediate cells had rounded euchromatic nuclei. The superficial cells were flattened with flat nuclei. All epithelial cells were connected together by many electron dense desmosomes, allowing narrow intercellular spaces. Under the epithelium, there was the Bowman's membrane which appeared as a thick collagen fibre layer (Fig. 7A, B). The stroma had regularly arranged collagen fibres parallel to the corneal surface. The keratocytes appeared as spindle-shaped cells with scanty cytoplasm and moderately electron dense oval nucleus. They appeared squeezed in-between collagen fibres (Fig. 7C). The Descemet's membrane displayed as homogenous acellular electron-dense layer and lined by a single layer of endothelial cell which appeared as flat cells having oval nuclei and their cytoplasm contained rough endoplasmic reticulum cisterna and mitochondria (Fig. 7D).

Group II (Latanoprost with BAC treated group), marked alterations including the various corneal layers were seen. The basal and intermediate epithelial cells appeared with cytoplasmic vacuoles, free ribosomes, distorted mitochondria, wide perinuclear space and shrunken nuclei (Fig. 8A, B). Moreover, the cells were separated by wide intercellular space (Fig. 8B). The superficial epithelial cells showed flat euchromatic nuclei with irregular contour. Wide intercellular spaces with partial loss of desmosomal junctions between some superficial epithelial cells were seen (Fig. 8C). The stroma exhibited keratocytes which were widely separated from the collagen fibres. The keratocytes appeared with cytoplasmic processes and their cytoplasm contained shrunken nuclei, dilated rough endoplasmic reticulum cisterna and lysosomes (Fig. 8D). Moreover, small areas in the stroma contained little amount of collagen fibres (Fig. 9A, B) and appearance of blood vessel was found (Fig. 9A). The endothelial cells had discontinued cell membrane and distorted mitochondria (Fig. 9B).

Group III (Recovery group) exhibited superficial epithelial cells with euchromatic nuclei and desmosomal junctions in between. Wide intercellular spaces with loss of desmosomes between some superficial epithelial cells were observed (Fig. 9C). The stroma exhibited regularly arranged collagen fibres. The keratocytes appeared with indented nucleus and abundant cytoplasm containing rough endoplasmic reticulum. Wide space between collagen fibres and keratocytes was 

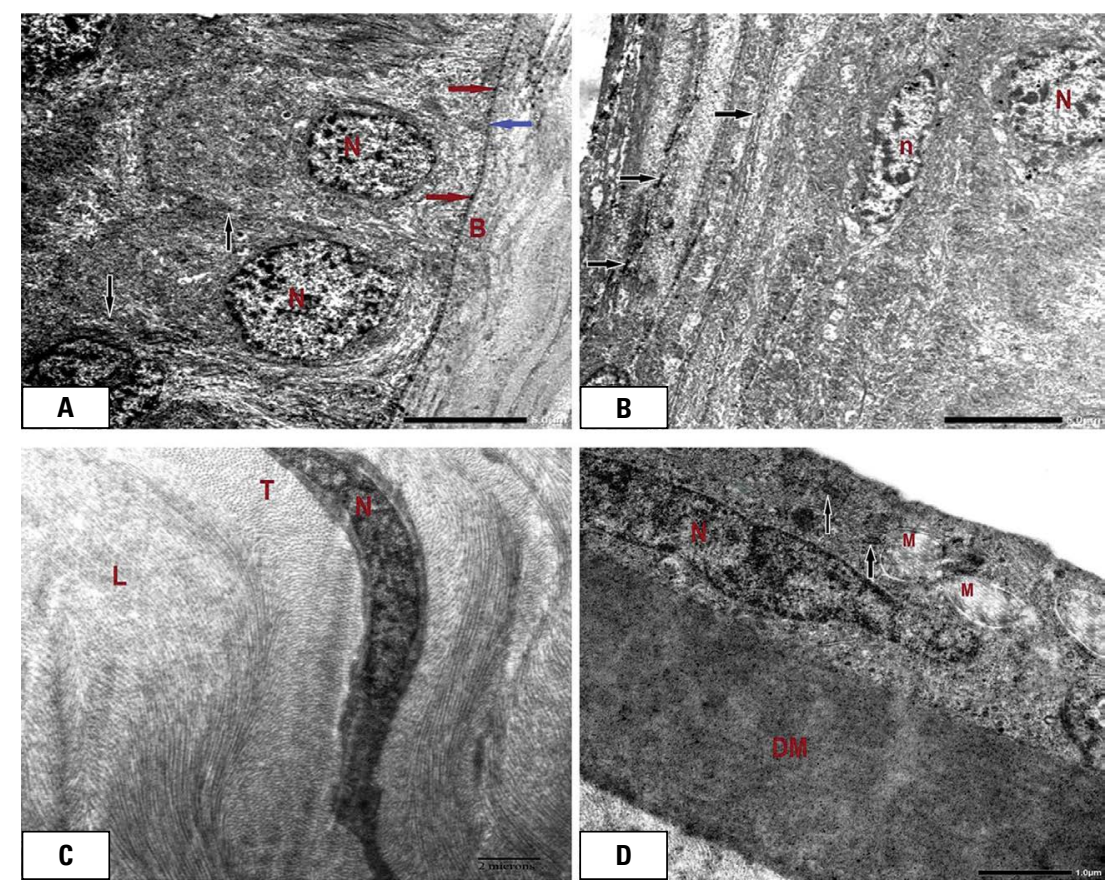

Figure 7. Electron micrographs of the cornea of control group illustrating: A. A basal columnar epithelial cells with euchromatic rounded nuclei (N) resting on clear intact Bowman's membrane (B). The epithelial cells are connected to each other by numerous desmosomes (black arrows) with narrow intercellular spaces. Notice, hemi-desmosomes appear as electron dense spots (red arrow) fixing the basal cells to the underlying basement membrane (blue arrow); $\times 4000$; B. The intermediate cells have rounded euchromatic nuclei (N). The superficial cells are flattened with flat nuclei (n). All epithelial cells are connected with each other by electron dense desmosomes (arrows); $\times 4000 ;$ C. A spindle-shaped keratocyte with scanty cytoplasm and moderately electron dense oval nucleus $(\mathrm{N})$ is seen squeezed between regularly arranged collagen fibres in longitudinal (L) and transverse (T) directions; $\times 5000$; D. A single endothelial cell layer with flat nucleus (N) and abundant cytoplasm containing mitochondria (M) and rough endoplasmic reticulum cisterna (arrows) is observed. The endothelial cells line thick homogenous electron-dense Descemet's membrane (DM); $\times 15000$.

observed (Fig. 9D). The Descemet's endothelial cells appeared with cytoplasmic vacuoles and distorted mitochondria (Fig. 10A).

Group IV (PF latanoprost treated group) showed corneal structure similar to that of the control group. The superficial epithelial cells were well organized with preserved desmosomal junctions (Fig. 10B). The stroma showed regularly arranged collagen fibres. The keratocytes with oval nucleus and rough endoplasmic reticulum in their cytoplasm were observed. The Descemet's endothelial cells appeared with flat nucleus and mitochondria (Fig. 10C).

\section{DISCUSSION}

Prostaglandin analogues are the first treatment used for glaucoma as a monotherapy or with other hypotensive agents. PGAs are preferable to other ocular hypotensive agents, because during long-term clinical use, there were no systemic side effects [16].

Among all the types of available PGAs, latanoprost was chosen in the present study, because this drug has good efficacy. Latanoprost is an esterified pro- drug of prostaglandin F2-alpha, more lipophilic than other PGAs, which means that it is better absorbed through the cornea. Latanoprost is well tolerated. So, patients receiving latanoprost treatment are more persistent than other ocular prostaglandins hypotensive medications [35]. Latanoprost is the first PGA that has an improved, recently produced formulation without BAC [1].

In this study, the histological changes within the corneal tissue with the use of currently available PG-derived eye drops, namely latanoprost (Xalatan) and PF PGA latanoprost (Monopost), in male guinea pigs were evaluated by light and electron microscope.

In the current study, the light microscopic corneal changes were confirmed by electron microscope. Corneal tissue from guinea pigs treated with latanoprost with BAC showed toxic changes that were considered to be related to the preservative, BAC, as previously reported by Trzeciecka et al. [38].

Epstein et al. [9] demonstrated that preservatives which exist in eye drops, such as BAC, change the permeability of the ocular surface epithelium, even 


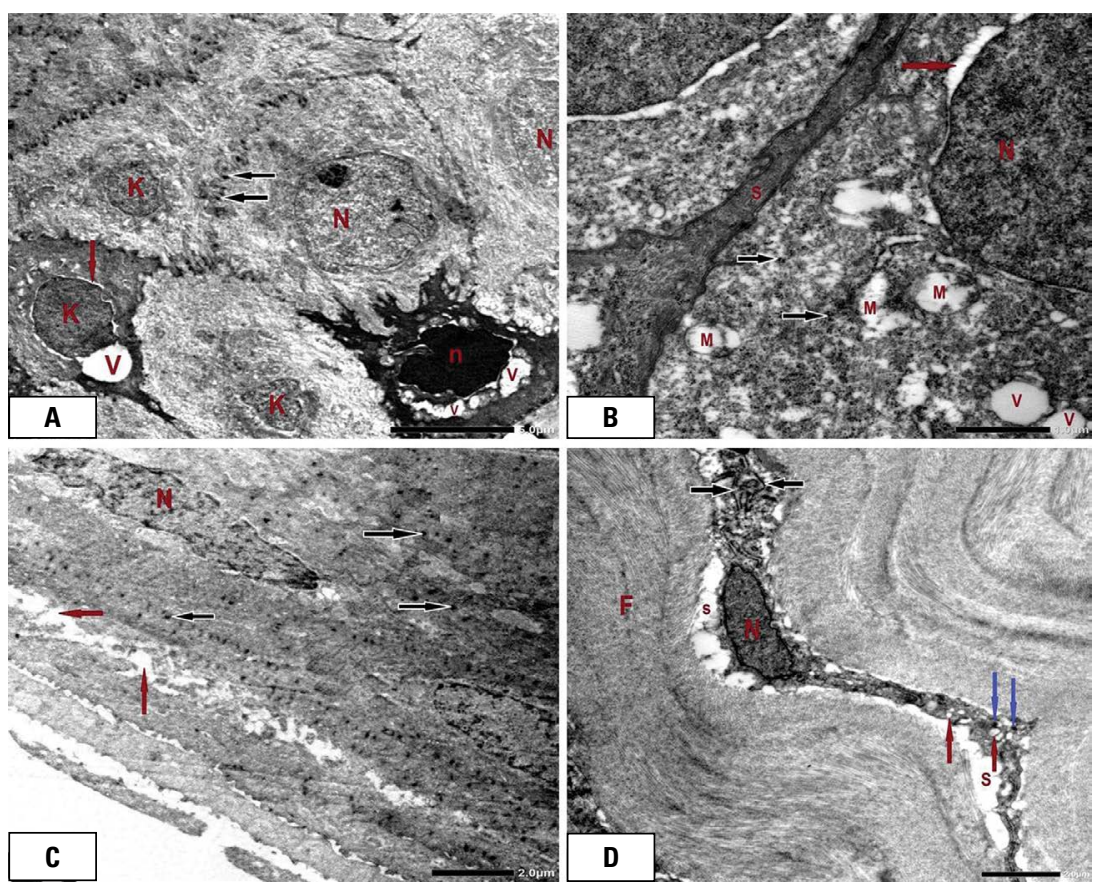

Figure 8. Electron micrographs of the cornea of group II illustrating: A. Basal and intermediate epithelial cells. One cell appears with cytoplasmic vacuoles (V) and condensed nuclei (n). Some cells have a euchromatic nuclei (N), whereas others have shrunken nuclei (K) and wide perinuclear space (red arrow). Notice, the cell membranes are interdigitated and connected by desmosomal junctions (black arrows); $\times 4000$; B. Epithelial cells with euchromatic nucleus (N) and wide perinuclear space (red arrow) are seen. The cytoplasm shows multiple vacuoles (V), free ribosomes (arrows) and distorted mitochondria (M). The cells are separated by wide intercellular space $(\mathrm{S}) ; \times 15000 ;$ C. The superficial epithelial cells have flat euchromatic nuclei (N) with irregular contour, some cells show preserved desmosomal junctions (black arrows). Notice, wide intercellular spaces with loss of junctions between some epithelial cells (red arrows); $\times 5000$; $\mathbf{D}$. The keratocyte has shrunken nucleus (N), variable sized cytoplasmic vacuoles (red arrows), dilated rough endoplasmic reticulum cisterna (black arrows) and lysosomes (blue arrow). The keratocyte is widely separated (S) from regularly arranged collagen fibres $(F)$ of stroma; $\times 5000$.

at very low doses. BAC gives rise to cell membranes lysis at the ocular surface.

Thinning of epithelial covering with desquamation of some surface cells was detected in the present study; this finding was confirmed morphometrically by showing highly significant decrease within the corneal epithelial thickness in comparison with the control animals. These results coincided with other authors $[10,22,39]$ who reported a decrease in the number of corneal epithelial layers with highly significant decrease in the central corneal thickness following long term treatment with BAC-containing eye drops. This might be due to degeneration of corneal epithelial cells with dead cells loss and failure of cellular regeneration and proliferation following long term treatment with BAC containing eye drops.

Guo et al. [13] and Soliman et al. [37] illustrated that the corneal epithelial cells desquamation with intracellular adenozyno-5'-trifosforan (ATP) depletion is accelerated by BAC. Among the various effects of ATP depletion, regulatory light chain of myosin II (MLC) phosphorylation has been detected. It is proven that the corneal epithelial cells exposure to BAC results in MLC phosphorylation that leads to contraction of the cytoskeleton of the epithelial cells leading to distortion the corneal barrier integrity.

Latanoprost with BAC-treated guinea pigs showed variable degrees of degeneration within the nuclei of the epithelial cells, endothelial cells and stromal keratocytes. This might be due to deoxyribonucleic acid (DNA) damage occurring in corneal cells after treatment of BAC-containing eye drops, as previously demonstrated with Kobayashi et al. [17] and Soliman et al. [37].

These results were also, in accordance with Barzilai and Yamamoto [4] and Baudouin et al. [5] who stated that BAC caused oxidative stress leading to free radicals generation, such as hydroxyl radicals and superoxide anion, and reactive oxygen species (ROS), such as singlet oxygen and hydrogen peroxide. Overproduction of ROS can induce cellular damage, particularly the DNA through the mitochondrial damage, which causes activation of endonuclease and apoptogenic proteases resulting in apoptosis. 

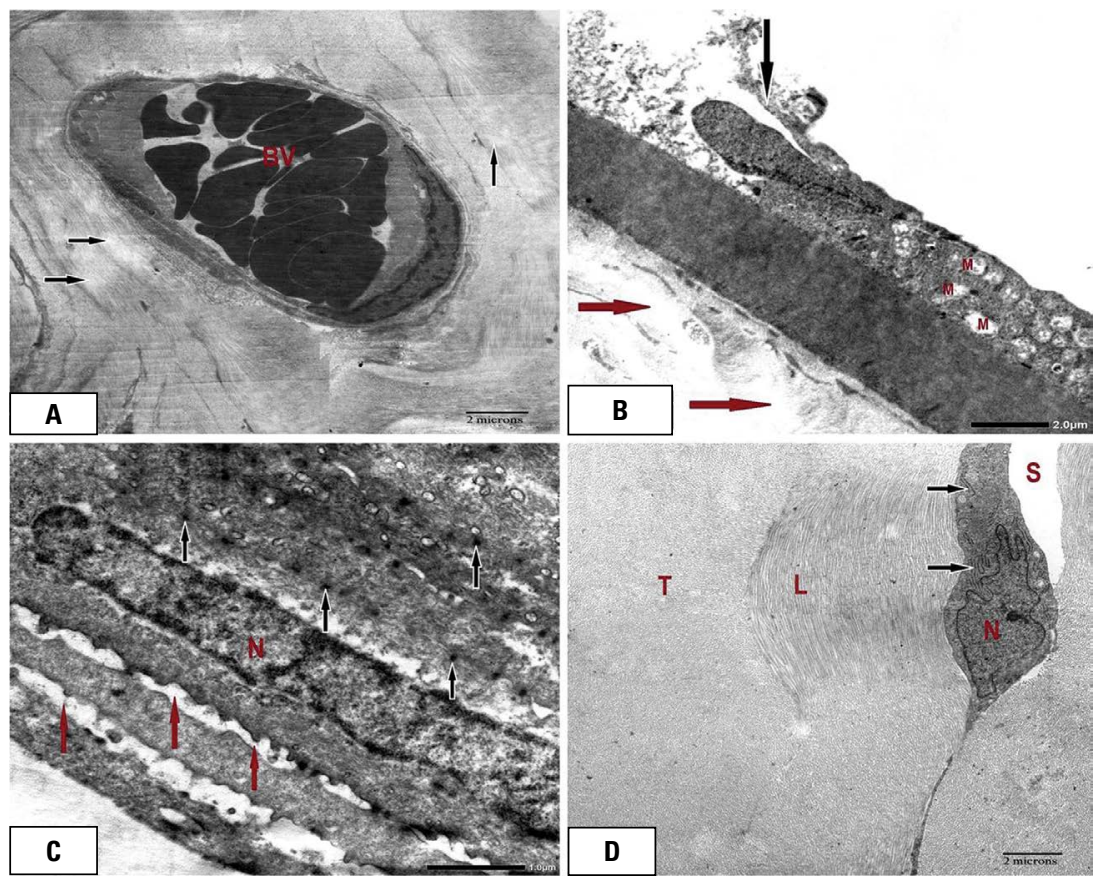

Figure 9. Electron micrographs of the cornea of group II (A, B) and group III (C, D); A. Group II: illustrating small areas in the stroma containing little amount of collagen fibres (black arrows) with appearance of blood vessel (BV); $\times 5000$; B. Group II: illustrating, discontinued endothelial cell membrane (black arrow) with distorted mitochondria (M). The stroma displays little amount of collagen fibres (red arrow); $\times 5000$; C. Group III: illustrating, superficial epithelial cells with flat euchromatic nuclei (N) and preserved desmosomal junctions in between (black arrows). Wide intercellular spaces between some superficial cells with loss of desmosomes are observed (red arrows); $\times 15000$; D. Group III: illustrating stroma with regularly arranged collagen fibres in longitudinal (L) and transverse (T) directions. The keratocyte appears with indented nucleus (N) and abundant cytoplasm containing rough endoplasmic reticulum cisterna (black arrow). Notice, wide space (S) between keratocyte and collagen fibres; $\times 5000$.
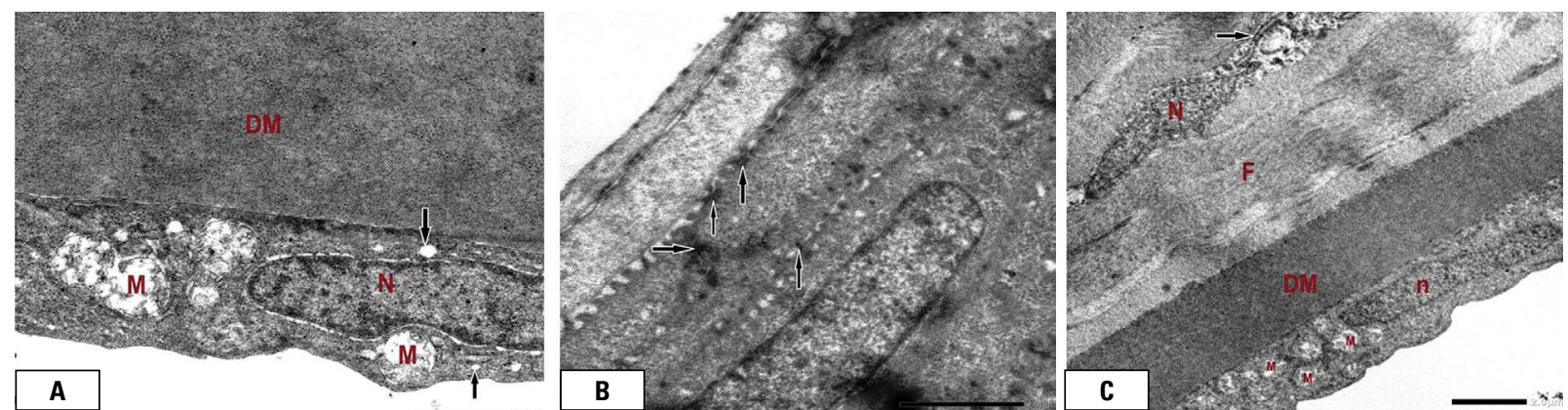

Figure 10. Electron micrographs of the cornea of group III (A) and group IV (B, C); A. Group III: Descemet's membrane (DM) lined by a single endothelial cell layer. The endothelial cell appears with flat nucleus $(\mathrm{N})$ and abundant cytoplasm containing distorted mitochondria (M) and vacuoles (black arrows); $\times 15000 ;$ B. Group IV: illustrating normal appearance of superficial epithelial cells with preserved desmosomal junctions (black arrows); $\times 5000 ;$ C. Group IV: illustrating stroma with regularly arranged collagen fibres (F). The keratocyte appears with oval nucleus ( $\mathrm{N}$ ) and rough endoplasmic reticulum (black arrow). The Descemet's membrane (DM) is lined by endothelial cells having flat nucleus ( $\mathrm{n}$ ) and mitochondria $(\mathrm{M}) ; \times 5000$.

Rosin and Bell [33] referred decreasing number of keratocytes within the stroma detected within corneal tissues from guinea pigs treated with latanoprost with BAC to their contraction, apoptosis and death of these cells after exposure to BAC. This finding was supported morphometrically as showing a highly significant decrease in the keratocytes number as compared with that of the control animals.

Disarrangement and loss of collagen fibres in some areas within the stroma in this study may be due to keratocytes loss as they synthesize and preserve stromal collagen; thus, keratocytes loss leads to 
less collagen secretion [23]. Another explanation of damaged corneal stromal cells following prolonged $B A C$ exposure was that PGAs are known to reduce IOP by upregulation of matrix metalloproteinases (MMPs), which are collagen degradation enzymes induced by prostaglandin F2-alpha [34, 39], causing connective tissue remodelling and degeneration of extracellular matrix, involving collagens of the sclera and ciliary muscle, resulting in lowering resistance of uveo-scleral outflow, thus improving aqueous humour outflow [18].

Stromal neovascularisation and congested blood vessels detected in the present study might be due to corneal stromal cells activation and migration to the site of injury following exposure to BAC. These stromal cells express vascular endothelial growth factor (VEGF) that induces angiogenesis with new blood vessels formation as previously explained by Mahmoud et al. [20].

It was also suggested by DeStafeno and Kim [8] that stromal neovascularisation might be due to unbalancing between angiogenic factors such as VEGF, fibroblast growth factor and anti-angiogenic ones such as angiostatin in the cornea.

Inflammatory cellular infiltration of the stroma detected within corneal sections from guinea pigs treated with latanoprost with BAC may be considered as a trial for healing and protection against infection which become easier with disturbance of the normal corneal epithelial barrier with prolonged treatment of BAC containing hypotensive eye drops.

Yang et al. [41] reported that inflammatory cellular infiltration of the stroma might be due to the expression of several inflammatory factors, such as chemotactic factors: monocyte chemo-attractant protein 1 , macrophage inflammatory protein-1 alpha and growth-regulated protein alpha, which increased after treatment with BAC within a week. These inflammatory factors have chemotactic activity for eosinophils monocytes and neutrophils, either separately or together.

Negri et al. [24] referred the inflammatory cellular infiltration of corneal stroma to the effect of BAC in lipoxygenase activation and secretion of inflammatory mediators, and multiple cytokines such as interleukin-la, tumour necrosis factor alpha, interleukin-8, leading to irritation and allergic reaction.

In the current study, widely separated irregularly arranged collagen fibres in Mallory's trichrome-stained corneal sections from guinea pigs treated with latanoprost with BAC might be due to degradation of its collagen fibres by active MMPs which are produced by prolonged exposure to BAC. These findings were in agreement with Gaton et al. [11] and Park et al. [26] who reported that PGAs cause up-regulation of MMPs that leads to degradation of extracellular matrix, including collagens of the ciliary muscle and sclera.

Another explanation of wide spaces in-between collagen fibres is that prolonged treatment with hypotensive eye drops containing BAC resulted in intracellular and extracellular oedema, as previously explained by Collin and Collin [7].

Histochemically, corneal sections of this group revealed mild positive PAS reaction in some parts and negative in other parts of the basement membrane. These results indicated reduction or even depletion of carbohydrates within the basement membrane of corneal epithelium.

Immunohistochemically, a marked immune positive cytoplasmic reaction for BAX in the covering epithelial cells and stromal keratocytes within corneal sections from guinea pigs treated with latanoprost with BAC was observed. This could be due to apoptosis of these cells. BAX is a nuclear-encoded protein that can penetrate the mitochondrial membrane and, thus, mediate cell death via apoptosis. So, increase reactivity for BAX indicates cellular apoptosis [40].

Guinea pigs left to recover without treatment for 2 weeks after treatment with latanoprost with BAC showed mild improvement of the histological changes of the cornea. However, some histological alterations were still persisting as desquamation of the superficial epithelial cells and corrugated Bowman's membrane. Small keratocytes with dark nuclei were noticed. Few endothelial cells appeared separated from the Descemet's membrane. These findings were in harmony with Soliman et al. [37] who demonstrated that there was mild recovery within the corneal sections of rats left for recovery after $0.02 \%$ BAC treatment with minimal decrease of the thickness of corneal epithelium and stromal degeneration.

On the other hand, light and electron microscopic studies of corneal sections from guinea pigs treated with PF latanoprost revealed nearly normal corneal structure. Moreover, these results were in harmony with Pauly et al. [27] and Yang et al. [41] who reported that rat corneal tissue presented better tolerance with PF latanoprost therapy. This improvement could be referred to absence of the BAC preservative from PGAs which in turn might improve the structure and function of the corneal epithelial barrier. 


\section{CONCLUSIONS}

Prostaglandins with BAC preservative derived eye drops result in many histological alterations in the corneal tissue. These alterations might be improved by withdrawal of the preservative. PF prostaglandin medication seems to be alternative to those preserved with BAC formulations.

\section{Conflict of interest: None declared}

\section{REFERENCES}

1. Alm A. Latanoprost in the treatment of glaucoma. Clin Ophthalmol. 2014; 8: 1967-1985, doi: 10.2147/OPTH. S59162, indexed in Pubmed: 25328381.

2. Ammar DA, Noecker RJ, Kahook MY. Effects of benzaIkonium chloride-preserved, polyquad-preserved, and sofZia-preserved topical glaucoma medications on human ocular epithelial cells. Adv Ther. 2010; 27(11): 837-845, doi: 10.1007/s12325-010-0070-1, indexed in Pubmed: 20931366.

3. Bancroft JD, Layton C. Theory and practice of histological technique. 7th ed. Churchill Livingstone, London 2010: 173-214.

4. Barzilai A, Yamamoto KI. DNA damage responses to oxidative stress. DNA Repair (Amst). 2004; 3(8-9): 1109-1115, doi: 10.1016/j.dnarep.2004.03.002, indexed in Pubmed: 15279799.

5. Baudouin $C$, Labbé $A$, Liang $H$, et al. Preservatives in eyedrops: the good, the bad and the ugly. Prog Retin Eye Res. 2010; 29(4): 312-334, doi: 10.1016/j.preteyeres.2010.03.001, indexed in Pubmed: 20302969.

6. Brignole-Baudouin F, Desbenoit N, Hamm G, et al. A new safety concern for glaucoma treatment demonstrated by mass spectrometry imaging of benzalkonium chloride distribution in the eye, an experimental study in rabbits. PLoS One. 2012; 7(11): e50180, doi: 10.1371/journal. pone.0050180, indexed in Pubmed: 23209668.

7. Collin SP, Collin HB. The corneal epithelial surface in the eyes of vertebrates: environmental and evolutionary influences on structure and function. J Morphol. 2006; 267(3): 273-291, doi: 10.1002/jmor.10400, indexed in Pubmed: 16323209.

8. DeStafeno JJ, Kim T. Topical bevacizumab therapy for corneal neovascularization. Arch Ophthalmol. 2007; 125(6): 834-836, doi: 10.1001/archopht.125.6.834, indexed in Pubmed: 17562998.

9. Epstein SP, Chen D, Asbell PA. Evaluation of biomarkers of inflammation in response to benzalkonium chloride on corneal and conjunctival epithelial cells. J Ocul Pharmacol Ther. 2009; 25(5): 415-424, doi: 10.1089/jop.2008.0140, indexed in Pubmed: 19857103.

10. Fasce F, Spinelli A, Bolognesi G, et al. Comparison of $B D$ Multivisc with the soft shell technique in cases with hard lens nucleus and Fuchs endothelial dystrophy. Eur J Ophthalmol. 2007; 17(5): 709-713, doi: 10.1177/112067210701700504, indexed in Pubmed: 17932844.

11. Gaton DD, Sagara T, Lindsey JD, et al. Increased matrix metalloproteinases 1,2 , and 3 in the monkey uveo- scleral outflow pathway after topical prostaglandin F(2 alpha)-isopropyl ester treatment. Arch Ophthalmol. 2001; 119(8): 1165-1170, doi: 10.1001/archopht. 119.8.1165, indexed in Pubmed: 11483084.

12. Glauert AM, Lewis PR. Biological specimen preparation for transmission electron microscope. 1st ed. Princeton University Press, London 1999.

13. Guo Y, Satpathy M, Wilson G, et al. Benzalkonium chloride induces dephosphorylation of Myosin light chain in cultured corneal epithelial cells. Invest Ophthalmol Vis Sci. 2007; 48(5): 2001-2008, doi: 10.1167/iovs.06-0613, indexed in Pubmed: 17460253.

14. Hae-Young L, Jie HK, Kyung ML, et al. Effect of prostaglandin analogues on tear proteomics and expression of cytokines and matrix metalloproteinases in the conjunctiva and cornea. Experimental Eye Res. 2011; 94(1): 13-21, doi: 10.1016/j.exer.2011.10.017.

15. Kalleny N, Soliman N. Light and electron microscopic study on the effect of topically applied hyaluronic acid on experimentally induced corneal alkali burn in albino rats. Egyptian J Histol. 2011; 34(4): 829-848, doi: 10.1097/01. ehx.0000407699.63226.2a.

16. Kim JH, Kim EJ, Kim YH, et al. In vivo effects of preservative-free and preserved prostaglandin analogs: mouse ocular surface study. Korean J Ophthalmol. 2015; 29(4): 270-279, doi: 10.3341/kjo.2015.29.4.270, indexed in Pubmed: 26240512.

17. Kobayashi N, Katsumi S, Imoto K, et al. Quantitation and visualization of ultraviolet-induced DNA damage using specific antibodies: application to pigment cell biology. Pigment Cell Res. 2001; 14(2): 94-102, doi: 10.1034/j.16000749.2001.140204.x, indexed in Pubmed: 11310797.

18. Lazcano-Gomez G, Ancona-Lezama D, Gil-Carrasco F, et al. Effects of topical travoprost $0.004 \%$ on intraocular pressure and corneal biomechanical properties in an animal model. Digit J Ophthalmol. 2016; 22(1): 1-5, doi: 10.5693/ djo.01.2015.03.001, indexed in Pubmed: 27330476.

19. Lee AJ, Goldberg I. Emerging drugs for ocular hypertension. Expert Opin Emerg Drugs. 2011; 16(1): 137-161, doi: 10.1517/14728214.2011.521631, indexed in Pubmed: 21352074.

20. Mahmoud B, Shady A, Meleegy UEl, et al. Effects of ultraviolet $B$ radiation on the cornea of adult male albino rats and the possible role of lornoxicam. Egypt J Histol. 2010; 33(1): 156-167, doi: 10.1097/00767537-201003000-00015.

21. Meda R, Wang Q, Paoloni D, et al. The impact of chronic use of prostaglandin analogues on the biomechanical properties of the cornea in patients with primary open-angle glaucoma. Br J Ophthalmol. 2017; 101(2): 120-125, doi: 10.1136/ bjophthalmol-2016-308432, indexed in Pubmed: 27162226.

22. Nada S, Elgendy M, Morcos M, et al. Histological study on conjunctival and corneal reactions in rabbits induced by chronic topical application of latanoprost and travoprost. Egyptian J Histol. 2013; 36(1): 139-148, doi: 10.1097/01. ehx.0000423477.48372.05.

23. Naroo S. Refractive surgery: a guide to assessment and management. 1st ed. Elsevier Health Sciences 2003: 96.

24. Negri L, Ferreras A, lester M. Timolol $0.1 \%$ in glaucomatous patients: efficacy, tolerance, and quality of life. J Ophthalmol. 2019; 2019: 4146124, doi: 10.1155/2019/4146124, indexed in Pubmed: 31191995. 
25. Okabe K, Kimura H, Okabe J, et al. Effect of benzalkonium chloride on transscleral drug delivery. Invest Ophthalmol Vis Sci. 2005; 46(2): 703-708, doi: 10.1167/iovs.03-0934, indexed in Pubmed: 15671302.

26. Park HL, Kim JH, Lee KM, et al. Effect of prostaglandin analogues on tear proteomics and expression of cytokines and matrix metalloproteinases in the conjunctiva and cornea. Exp Eye Res. 2012; 94(1): 13-21, doi: 10.1016/j. exer.2011.10.017, indexed in Pubmed: 22067128.

27. Pauly $A$, Roubeix $C$, Liang $H$, et al. In vitro and in vivo comparative toxicological study of a new preservative-free latanoprost formulation. Invest Ophthalmol Vis Sci. 2012; 53(13): 8172-8180, doi: 10.1167/iovs.12-10766, indexed in Pubmed: 23150620.

28. Peat J, Barton B. Medical statistics. A Guid to data analysis and critical appraisal. 1st ed. Wiley-Blackwell 2005: 113-119.

29. Penault-Llorca F, Bouabdallah R, Devilard E, et al. Analysis of BAX expression in human tissues using the anti-BAX, 4F11 monoclonal antibody on paraffin sections. Pathol Res Pract. 1998; 194(7): 457-464, doi: 10.1016/s03440338(98)80114-3, indexed in Pubmed: 9728362.

30. Quigley HA, Broman AT. The number of people with glaucoma worldwide in 2010 and 2020. Br J Ophthalmol. 2006; 90(3): 262-267, doi: 10.1136/bjo.2005.081224, indexed in Pubmed: 16488940.

31. Quigley H. Glaucoma. Lancet. 2011; 377(9774): 1367-1377, doi: 10.1016/s0140-6736(10)61423-7.

32. Ramli N, Supramaniam G, Samsudin A, et al. Ocular surface disease in glaucoma: effect of polypharmacy and preservatives. Optom Vis Sci. 2015; 92(9): e222-e226, doi: 10.1097/OPX.0000000000000542, indexed in Pubmed: 25730335.

33. Rosin LM, Bell NP. Preservative toxicity in glaucoma medication: clinical evaluation of benzalkonium chloride-free $0.5 \%$ timolol eye drops. Clin Ophthalmol. 2013; 7: 2131-2135, doi: 10.2147/OPTH.S41358, indexed in Pubmed: 24204115.
34. Saika S, Ohnishi Y, Ooshima A, et al. Epithelial repair: roles of extracellular matrix. Cornea. 2002; 21(2 Suppl 1): S23-S29, doi: 10.1097/00003226-200203001-00006, indexed in Pubmed: 11995806.

35. Shafranov G. Comparing prostaglandins. Glaucoma Today. 2005: 37-39.

36. Smith M. Medication review: prostaglandin analogs for glaucoma. Pharmacy Times. 2015; 19(6): 949-956.

37. Soliman ME, Mahmoud BL, Kafafy MA. Histological changes in cornea following repeated exposure to BenzalkoniumChlorid and the possible protective effect of topically applied sodium hyaluronate. Nature Science. 2015; 13(5): 64-76.

38. Trzeciecka A, Paterno JJ, Toropainen E, et al. Long-term topical application of preservative-free prostaglandin analogues evokes macrophage infiltration in the ocular adnexa. Eur J Pharmacol. 2016; 788: 12-20, doi: 10.1016/j.ejphar.2016.06.014, indexed in Pubmed: 27288881.

39. Uzunel UD. The effect of prostaglandin analogues on central corneal thickness. Int J OphthalmolClin Res. 2018; 5(1), doi: 10.23937/2378-346x/1410084.

40. Westphal D, Dewson G, Czabotar PE, et al. Molecular biology of Bax and Bak activation and action. Biochim Biophys Acta. 2011; 1813(4): 521-531, doi: 10.1016/j. bbamcr.2010.12.019, indexed in Pubmed: 21195116.

41. Yang Q, Zhang Y, Liu X, et al. A comparison of the effects of benzalkonium chloride on ocular surfaces between C57BL/6 and BALB/C mice. Int J Mol Sci. 2017; 18(3), doi: 10.3390/ijms18030509, indexed in Pubmed: 28245636.

42. Yee RW, Norcom EG, Zhao XC. Comparison of the relative toxicity of travoprost $0.004 \%$ without benzalkonium chloride and latanoprost $0.005 \%$ in an immortalized human cornea epithelial cell culture system. Adv Ther. 2006; 23(4): 511-519, doi: 10.1007/BF02850039, indexed in Pubmed: 17050493. 